\section{Rheumatoide Arthritis: IL-6-Serumspiegel sagt Therapieansprechen voraus}

Boyapati A et al. Association of High Serum Interleukin-6 Levels With Severe Progression of Rheumatoid Arthritis and Increased Treatment Response Differentiating Sarilumab From Adalimumab or Methotrexate in a Post Hoc Analysis. Arthritis Rheumatol 2020; 72:

1456-1466. doi: 10.1002/art.41299

Interleukin-6 (IL-6) spielt eine wichtige Rolle bei der Pathogenese der rheumatoiden Arthritis (RA). Mittlerweile sind gegen den IL-6-Signalweg gerichtete monoklonale Antikörper (z. B. Sarilumab) zur Therapie der RA zugelassen. Lässt sich anhand des initialen IL-6-Serumspiegels das Therapieansprechen voraussagen? Dieser Frage ging ein Team internationaler Wissenschaftler mithilfe einer post hocAnalyse von Daten zweier Phase III-Studien nach.

Im Rahmen der MONARCH-Studie wurden Patienten mit mäßig bis schwerer RA und Methotrexat-Unverträglichkeit bzw. schlechtem Ansprechen auf diese Therapie über 24 Wochen mit Sarilumab oder Adalimumab behandelt, erläutern die Forscher. Im Rahmen der MOBILITY-Studie wurden Patienten mit mäßig bis schwerer RA und inadäquatem Methotrexat-Ansprechen über 52 Wochen mit 2 verschiedenen Sarilumab-Dosen bzw. Placebo in Kombination mit Methotrexat behandelt. Von allen Patienten standen vor Studienbeginn entnommene Blutproben zur Verfügung, anhand welcher die Wissenschaftler die Serum-IL-6-Konzentration bestimmten. Anschließend prüften sie, inwiefern sich die verschiedenen IL-6-Terzile bezüglich des Therapieansprechens auf Sarilumab unterschieden.

\section{Ergebnisse}

Die Auswertung der MONARCH-Daten ergab: Die Patienten des höchsten IL-6-Terzils ( $\geq$ das Dreifache des oberen Normwerts zu Studienbeginn; $n=100$ ) wiesen im Vergleich zu den Patienten des niedrigsten Terzils initial eine höhere Krankheitsaktivität auf. Das Ausmaß der klinischen Besse- rung im Verlauf der 24-wöchigen Sarilumab-vs. Adalimumab-Behandlung war bei Patienten mit hohem Ausgangs-IL6-Spiegel größer als bei Patienten mit niedrigem Spiegel. Die Analyse der MOBILITYStudie zeigte: Im Vergleich zu Patienten mit niedrigem IL-6-Spiegel ( $\mathrm{n}=397)$ wiesen Patienten mit hohem IL-6-Spiegel $(n=398)$ initial eine höhere Krankheitsaktivität sowie ausgeprägtere Gelenkschäden auf und erlitten zudem häufiger einen Progress der Gelenkschäden. Ferner erfuhren sie im Verlauf der 52-wöchigen Therapiephase eine geringere klinische Verbesserung. In beiden Studien stellten der IL6- und der CRP-Spiegel bei Studienbeginn Prädiktoren bezüglich des Behandlungserfolgs dar. Im Hinblick auf das Sicherheitsprofil unterscheiden sich die Patienten der 3 IL-6-Terzile in beiden Studien nicht wesentlich.

\section{FAZIT}

Bei RA-Patienten mit unzureichendem Methotrexat-Ansprechen oder -Unverträglichkeit, so das Fazit der Autoren, stellt der IL-6-Serumspiegel möglicherweise einen Prognosemarker im Hinblick auf den Krankheitsprogress sowie die Erkrankungsschwere dar. Sie gehen davon aus, dass Patienten mit hohem IL-6-Spiegel von Sarilumab im Vergleich zu Adalimumab oder Methotrexat profitieren. Prospektive Studien müssen nun ihrer Ansicht nach diese post hoc-Analyseergebnisse bestätigen.

Dr. med. Judith Lorenz, Künzell 